\title{
CENTROS CULTURAIS - A IMPORTÂNCIA DO EDIFÍCIO PARA DIVULGAÇÃO DA CULTURA. UMA PROPOSTA PARA A CIDADE DE PRESIDENTE BERNARDES/SP.
}

Bruna de Andrade Ferreira, Fabrícia Dias da Cunha de Moraes Fernandes Borges

Universidade do Oeste Paulista - UNOESTE, curso de Arquitetura e Urbanismo, Presidente Prudente, SP. E-mail: bruna andrade.f@hotmail.com

\begin{abstract}
RESUMO
O seguinte trabalho ressalta a origem dos espaços culturais, advinda da ideia de instituições criadas anteriormente como a biblioteca, o teatro e o museu. Apresenta também exemplos de espaços culturais importantes para a história e influenciadores de muitos outros que surgiram após suas criações. O objetivo do trabalho busca enfatizar que o centro cultural trata-se de um espaço dinâmico e pertencente à cidade, isto é, espaço público e político onde seus usuários possuem total liberdade de expressão; deve ser o local da cultura viva, que permita a formação de uma consciência sobre a realidade. $O$ trabalho se desenvolveu por meio de pesquisa bibliográfica, dentre eles livros, artigos, teses principalmente com metodologia qualitativa, bem como de dados levantados em campo referente ao estudo de caso da antiga Estação Ferroviária da cidade de Presidente Bernardes/SP.

Palavras-chave: Centro cultural; Espaços culturais; Espaço Público; Estação Ferroviária; Presidente Bernardes/SP.

\section{CULTURAL CENTERS - THE IMPORTANCE OF THE EDUCATION FOR THE DISCLOSURE OF CULTURE.} A PROPOSAL FOR THE CITY OF PRESIDENT BERNARDES/SP.
\end{abstract}

\begin{abstract}
The following work highlights the origin of the cultural spaces, coming from the idea of institutions created previously like the library, the theater and the museum. It also presents examples of cultural spaces important to history and influencers of many others that have emerged after their creations. The objective of this work is to emphasize that the cultural center is a dynamic space belonging to the city, that is, public and political space where its users have total freedom of expression; must be the place of living culture, which allows the formation of an awareness of reality. The work was developed through bibliographic research, among them books, articles, theses mainly with qualitative methodology, as well as data collected in the field referring to the case study of the former Railway Station of the city of Presidente Bernardes / SP.
\end{abstract}

Keywords: Cultural center; Cultural spaces; Public place; Railway Station; Presidente Bernardes/ SP.

\section{INTRODUÇÃO}

A cultura é a vida de um povo. Seguindo a visão de Hall (2006), trata-se de padrões comportamentais adquiridos com a experiência e vivência em sociedade. Trata-se de uma herança social onde a relação de pertença perpetua com a educação formal, informal e não formal através de um ambiente construído com o tempo, com as mudanças e as necessidades das pessoas.

Na segunda metade do século XX, de acordo com Ramos (2007), o termo centro cultural passou a ser discutido e implantado nos grandes países da Europa, que buscavam como propósito democratizar a cultura para além das tendências da cultura de massa e, desta forma tiveram seus exemplos copiados por muitos outros países. 
Assim como Ramos (2007), Milanesi (2003) pondera que a ideia dos centros culturais relaciona-se a outras instituições criadas anteriormente, como por exemplo a biblioteca, sendo está considerada a mais antiga das criações. Também os teatros, os cinemas e os museus, espaços estes que de certa forma, influenciaram a criação dos centros culturais que se trata justamente de um espaço que engloba atualmente todas estas referências.

No Brasil, Milanesi (2003) afirma que a vida cultural no país foi formada pelas tradições trazidas pelos colonizadores, mais especificamente com a vinda da Corte Portuguesa, que trouxeram consigo o modelo de se projetar bibliotecas, teatros e museus. E que juntos ou isoladamente, são identificados como centros de cultura, tratando-se de espaços semelhantes, porém, com nomes distintos.

Historicamente, de acordo com Miranda (apud VAINER; FERRAZ, 2017, p. 5), "na virada para o século XX, a Revolução Industrial acarretou diversas mudanças em questões sociais e urbanas com a chegada das indústrias". A sociedade passou a partir daí por um processo da perda de referências frente à redução e o esquecimento dos espaços de convívio social.

Atualmente, constatou-se a importância dos espaços voltados a cultura, a criação destes aprimoram não só o ambiente em que se insere, como também a vida de seus usuários. Para tanto, o objetivo deste trabalho busca esclarecer a importância de tal espaço criado para uma sociedade que prioriza a democratização de seu uso desde os primeiros tempos, como também une e constrói uma cidadania.

\section{METODOLOGIA}

Explorar as problemáticas em relação a cultura, os espaços culturais e a sociedade em si, foram a tônica desta pesquisa, especificamente dos dados obtidos através da pesquisa a campo referente ao estudo de caso da Estação Ferroviária da cidade de Presidente Bernardes/SP, bem como através de dados coletados por meio de livros, artigos e teses especializadas referentes o assunto proposto, sobretudo, à metodologia qualitativa de pesquisa. Para Turato (2005), as pesquisas que utilizam o método qualitativo devem trabalhar com valores, crenças, representações, hábitos, atitudes e opiniões, que busca compreender o significado que o acontecimento tem para pessoas comuns, em situações particulares, enfatizando-se a importância da interação simbólica e da cultura para a compreensão do todo.

\section{RESULTADOS}

Presidente Bernardes teve seu projeto urbano traçado em 1920 pelo Cel. José Soares Marcondes, em expansão a linha tronco da estrada de ferro Sorocabana. A estrada de ferro como a própria história do município relata, foi o principal meio fundador e está localizada no centro da cidade em conjunto com a Praça da Bandeira, próxima ao ponto de maior concentração de pessoas e atividades. Rodeada pelo comércio central, isola-se e destaca-se como um monumento histórico e cultural. Apesar da carga histórica da cidade, a estação é um dos poucos edifícios que restaram com significado arquitetônico relevante.

FIGURA 01 - Atual estado da Estação Ferroviária de Presidente Bernardes/SP
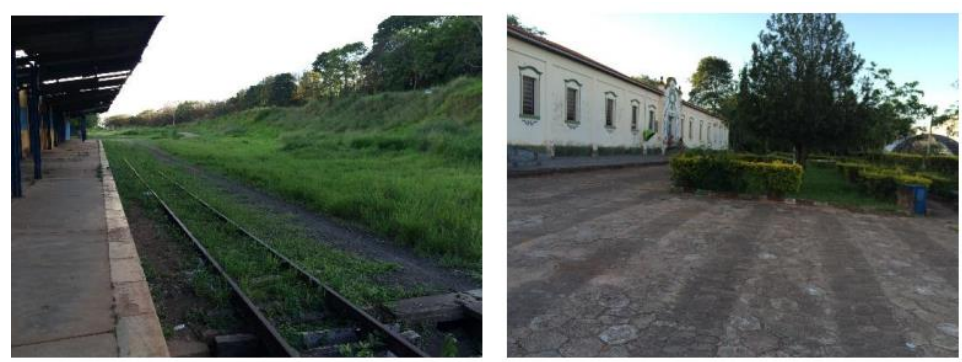

Fonte: Autora (2016). 
Segundo Lima et al. (2014) apud Giesbrecht (2014), a estação está desativada desde 1990, onde passa a ser um local esquecido e abandonado. Contudo, no ano 2000 ela ressurge com enfoque na importância cultural mesmo com as inadequadas transformações e intervenções do ponto de vista arquitetônico e de restauração, dando abrigo ao novo uso da biblioteca municipal, mantida até o presente momento. Porém, mesmo com a proposta da biblioteca para o local, não foi solucionado os problemas de abandono público. O local permanece com seu uso e entorno imediato em total degradação e descuido, tanto pelos usuários, bem como do poder público.

FIGURA 02 - Abandono, degradação e vandalismo, entorno imediato da Estação Ferroviária de Presidente Bernardes/SP.
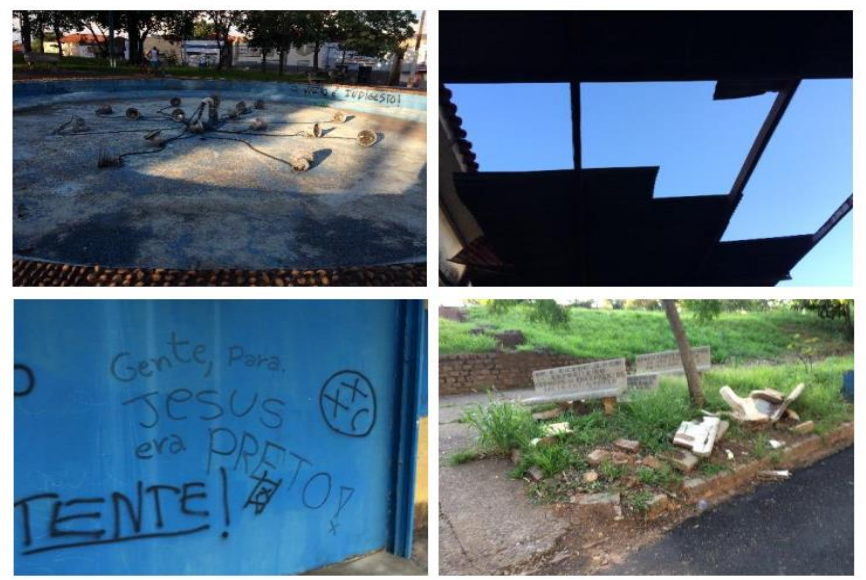

Fonte: Autora (2016).

Entre os setores da cidade de Presidente Bernardes, o mais prejudicado é o cultural, pela falta de estrutura fornecida pela cidade, como também pela falta do incentivo daqueles que têm o poder de mudar essa situação.

A proposta de um Centro Cultural para a cidade de Presidente Bernardes, implantado em conjunto à antiga linha férrea da cidade, deve potencializar questões históricas e culturais, proporcionar vida novamente ao espaço, visto como uma excelente oportunidade de ligar a linha férrea a praça da Bandeira, sendo está a principal praça da cidade e encontra-se em estado de deterioração.

FIGURA 03 - Proposta requalificação e edifício cultural

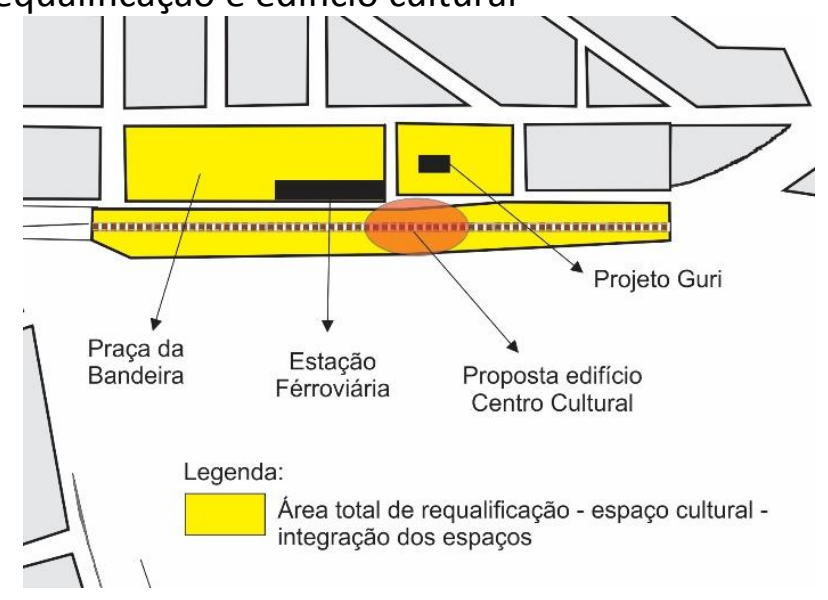

Fonte: Autora (2017).

Para as diretrizes, no que se diz respeito aos edifícios já construídos, a proposta de requalificação desses espaços e de seu entorno proporcionaria novos usos para o local, onde 
consequentemente ocorrerá maior utilização destes espaços, como também a valorização e preservação do edifício da antiga Estação Ferroviária.

Se tratando dos espaços abandonados e deteriorados, a proposta de requalificação levaria à utilização dos usuários, onde a permanência nestes espaços, agrega diversos fatores como a segurança, qualidade de vida social, turismo e preserva a identidade e memória da cidade ao se tratar de um espaço histórico com grandes valores culturais.

\section{DISCUSSÃO}

Atualmente, conforme Silva (1995), muitas instituições têm sido criadas com a denominação de centros culturais ou outras semelhantes, tais como: casas de cultura, centros de cultura, espaços culturais, etc. Em sua essência, o termo centro cultural é bastante recente e não está completamente definido. Historicamente, a origem dos espaços culturais teve influências de instituições criadas anteriormente, entre os principais: a biblioteca, o teatro e os museus.

A Biblioteca de Alexandria a mais antiga da história, segundo Silva (1995), era constituída por um espaço cultural formado por palácios reais que possuíam em seus acervos diversos tipos de documentos, com o objetivo de preservar a existência histórica da Grécia Antiga nos campos da religião, mitologia, astronomia, filosofia, medicina, zoologia, geografia, etc. O local funcionava como um espaço de estudos junto a um local de culto às divindades que armazenava estátuas, obras de arte, instrumentos cirúrgicos e astronômicos, peles de animais raros, presas de elefantes, pedras e minérios trazidos de terras distantes. O complexo dispunha também um anfiteatro, um observatório, salas de trabalho, refeitório, jardim botânico e zoológico.

FIGURA 04 - Projeção da Biblioteca de Alexandria

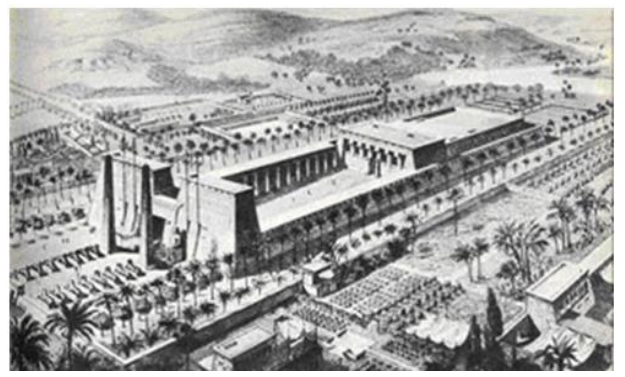

Fonte: http://www.walterjorge.com/novo/pages/viagens---egito---35---biblioteca-de-alexandria.php.

Se tratando de arquitetura, para Ferraz (apud VAINER, 2017, p. 121), foi no ano de 1982 que uma obra revolucionaria esta questão do espaço especifico para o lazer, com a criação de uma obra internacional, advinda da França, o projeto do edifício Cultural Georges Pompidou abriu fronteiras para o mundo inteiro da sua importância para a sociedade e arquitetura, com mudanças bruscas de escala, e estranheza para a sua vizinhança.

Para complementar, Milanesi (2003) diz que o projeto do Centro Cultural Georges Pompidou, teve seu projeto inicial lançado em 1971 conforme uma iniciativa do governo francês que promoveu um concurso internacional de arquitetura com o objetivo da construção do centro cultural, onde o projeto vencedor pertence a dupla de arquitetos anglo-italiana Rogers e Piano. 
FIGURA 05 - Centro Cultural Georges Pompidou

Fonte: https://www.centrepompidou.fr/fr/Le-Centre-Pompidou/L-histoire.

\section{O SURGIMENTO DOS ESPAÇOS CULTURAIS NO BRASIL}

De acordo com a linha de pensamento de Coelho (2001), as cidades brasileiras seguiam uma certa ordem referente as implantações dos edifícios disseminadores de cultura: primeiro as cidades sonhavam com uma biblioteca, depois com o teatro e mais tarde com um cinema e, em seguida foi a vez dos museus.

No Brasil, o primeiro teatro foi inaugurado em 1911, trata-se do Teatro Municipal de São Paulo, que como salientado por Milanesi (2003), era considerado o maior edifício já construído até então na cidade, portanto, referência da riqueza paulista. Em outras palavras, a finalidade dos museus era de preservar a história do local em que se inseria. Os cinemas por sua vez tiveram sua expansão dentro dos teatros, onde ainda segundo o autor, define que a partir daí surgiu a expressão nos anos de 1920 e 1930 o cine-teatro.

Historicamente, de acordo com Miranda (apud VAINER; FERRAZ, 2017, p. 5), na virada para o século XX, a revolução industrial acarretou diversas mudanças em questões sociais e urbanas com a chegada das indústrias. A sociedade passa a partir daí por um processo da perda de referências frente à redução e o esquecimento dos espaços de convívio social. Em São Paulo, por exemplo, com as diversas transformações advindas deste setor industrial, do emprego e da falta de tempo para o lazer, estudiosos e principalmente pessoas ligadas ao meio das artes se revolucionaram, buscando solucionar esta questão da retomada do convívio social e da criação de espaços voltados ao culto do lazer e da cultura para uma sociedade como um todo.

Referindo-se aos centros culturais no Brasil, os primeiros espaços destinados à esta função surgiram na década de 1980, na cidade de São Paulo, como afirma o autor Ramos (2007), o Centro Cultural do Jabaquara e o Centro Cultural São Paulo, ambos de iniciativa do Governo do Estado.

O Sesc Pompeia, por exemplo, localizado em São Paulo, surgiu através de ações socioeducativas, movimento este que teve início no ano de 1982, que priorizava a preservação do patrimônio arquitetônico. O programa de necessidades para o Sesc Pompéia, segundo Vainer e Ferraz (2016), deveria fomentar a convivência entre as pessoas como fórmula infalível de produção cultural, bem como incentivar o esporte recreativo. A ideia era reforçar e fomentar a recreação, o esporte leve, assim programa e projeto arquitetônico se fundiram.

FIGURA 06 - Sesc Pompéia

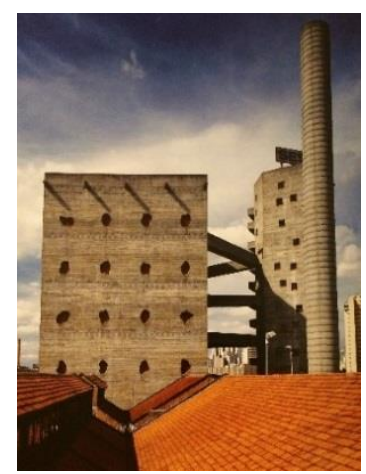


Fonte: Livro "A Cidadela da Liberdade - Lina Bo Bardi e o Sesc Pompeia", 2016, p. 124.

Ramos (2007) destaca a importância da política para a criação dos centros culturais como espaços democráticos e de acesso a todos, dizendo que as disseminações dos centros culturais no Brasil dependem muito do investimento do poder público, principalmente em questões de fiscalização desses espaços.

\section{CONCLUSÃO}

Explorar as problemáticas em relação a cultura, patrimônio e espaços históricos, constatou-se no decorrer do desenvolvimento desta pesquisa, a capacidade de melhoria através de intervenções urbanas, por meio de um projeto de um edifício de caráter público, cultural e educativo, busca gerar um espaço de lazer para a população.

Portanto, podemos concluir que um centro cultural como lugar público e político é fundamental para a democratização de uma sociedade, um local que amplia conhecimentos diversos e se torna referencial em questões de turismo e lazer para uma localidade, pois com a criação deste, vários fatores de melhorias urbanísticas surgiram em conjunto a favor da cidade. Além de servir como um ponto de encontro onde as pessoas possam trocar ideias, serve também para se tratar de questões emergentes e atuais da cidade. E o mais importante é que neste espaço as pessoas têm total liberdade de expressão.

\section{REFERÊNCIAS BIBLIOGRÁFICAS}

COELHO, Teixeira. O que é ação cultural / Teixeira Coelho. - São Paulo : Brasiliense, 2001. (Coleção primeiros passos ; 216).

HALL, Stuart. A identidade cultural na pós-modernidade. 10 ed. Rio de Janeiro: DP \& A, 2005. 102 p.

MILANESI, L. A casa da invenção. 3. ed., ver. e ampl. São Caetano do Sul, SP: Ateliê Editorial, 1997.

RAMOS, Luciene Borges. 0 centro cultural como equipamento disseminador de informação: um estudo sobre a ação do Galpão Cine Horto. 246f. Dissertação (Mestrado em Ciência da Informação) - Escola de Ciência da Informação, Universidade Federal de Minas Gerais. Belo Horizonte, 2007.

SANTOS, W. Presidente Bernardes - História de sua fundação. Presidente Prudente: Jornalismo e Pesquisa Histórica, 2000.

SILVA, José Afonso da. Ordenação constitucional da cultura. São Paulo: Malheiros Editores, 2001.

VAINER, André; FERRAZ, Marcelo. Cidadela da liberdade: Lina Bo Bardi e o Sesc Pompeia / Organização de André Vainer e Marcelo Ferraz. - São Paulo: Edições Sesc São Paulo, 2016. - 168 p.: il. Fotografias; edição bilíngue (português/inglês).

WIDMER, Ernst. Problemas da Difusão Cultural. In: Cadernos de Difusão Cultural da. Salvador: UFBA - Universidade Federal da Bahia, 1979. 